\title{
Approximating the Dirac Distribution for Fourier Analysis
}

\author{
Stuart B. Cohen and Ivan N. Kirschner \\ Department of Naval Architecture and Marine Engineering, \\ The University of Michigan, Ann Arbor, Michigan 48109
}

Received July 11, 1989; revised November 21, 1989

\begin{abstract}
For some boundary or initial value problems, the presence of a Dirac distribution on the boundary or in the field results in finite solutions at some points in the domain. However, its presence leads to difficulties if the problem is solved analytically using a Fourier decomposition, since computation and presentation of the solution usually necessitate some sort of truncation. To circumvent this problem, the Dirac distribution is olten approximated by a Gaussian distribution, which results in a very simple Fourier transform on an infinite domain. On a finite domain the transform is not as simple, but may still be computed. However, the derivative of the Gaussian is discontinuous on the finite domain, since the smooth function has been truncated. Thus a different approximation, the $\beta_{\pi}$-dstribution is proposed. This function satisfies the same criteria which make the Gaussian applicable as an approximation of the Dirac distribution on the infinite domain, but its derivative is continuous everywhere on the finite domain. This article presents a procedure for computing the Fourier coefficients of the $\beta_{\pi}$-distribution. Since a large value of the order of the distribution is chosen to approximate the singular behavior, the integral for the Fourier coefficients must be evaluated using a Fourier-Bessel decomposition, which allows the computation to be carried out over large values of the Fourier index. The technique is illustrated with application to a simple twodimensional boundary value problem containing a singularity in the boundary condition. Convergence is significantly improved if the proposed distribution is used. Values of some Fourier coefficients of the $\beta_{\pi}$-distribution are provided in an appendix for several values of its order. (C) 1991 Acadernic Press, Inc
\end{abstract}

\section{INTRODUCTION}

Consider the problem of determining the temperature distribution in a solid unit disk resulting from a flame supplying heat at the point $(x, y)=(1,0)$ on the boundary, which is otherwise maintained at temperature zero. This boundary value problem can be stated:

$$
\begin{aligned}
\nabla^{2} \omega(r, \theta) & =0 \\
\omega(1,0) & =\delta_{0}(0) \\
\omega(r, \theta) & =\omega(r, \theta+2 \pi n),
\end{aligned}
$$


Where $n$ is a non-negative integer and $\delta_{0}(\theta)$ is the Dirac distribution on domain $\theta$ with source point $\theta=0$. This generalized function has the properties:

$$
\begin{gathered}
\delta_{0}(\theta)=0 \quad \forall \theta \neq 0 \\
\int_{\theta=-\pi}^{\pi} f(\theta) \delta_{0}(\theta) d \theta=f(0)
\end{gathered}
$$

for any function continuous and bounded on $\theta \in[-\pi, \pi]$.

As is well known, the Fourier series solution of a similar problem with general boundary conditions is [13]

$$
\omega(r, \theta)=\frac{a_{0}}{2}+\sum_{n=1}^{\infty}\left[a_{n} \cos (n \theta)+b_{n} \sin (n \theta)\right] r^{n}
$$

where the Fourier coefficients, $a_{n}$ and $b_{n}$, are

$$
\begin{aligned}
& a_{n}=\frac{1}{\pi} \int_{\theta=-\pi}^{\pi} f(\theta) \cos (n \theta) d \theta \\
& b_{n}=\frac{1}{\pi} \int_{\theta=-\pi}^{\pi} f(\theta) \sin (n \theta) d \theta .
\end{aligned}
$$

It has been verified, [9], that the Fourier series solution with the singular boundary condition, Eq. (2), has coefficients

$$
\begin{aligned}
& a_{n}=1 / \pi \\
& b_{n}=0
\end{aligned}
$$

and converges everywhere in the domain and on the boundary, except at the source point. A similar problem can be posed on a domain which is of infinite extent in one dimension, say $x \in(-\infty, \infty), y \in[-1,1]$. In this case the solution involves a Fourier transform in $x$.

The existence of analytic solutions of such singular problems is well knowr. However, actual computation of the solutions is problematic, since the series must always be truncated. The resulting error is obviously infinite at the source point, since any truncated series has a finite value at that point. While the error is inite at all other points in the domain and on the boundary, it is not at all uniform throughout, since it increases drastically near the source point. The resulting approximation is unsatisfactory.

Similar problems result when computing frequency information from a time history of finite duration using a Fourier transform. The analogous result is unacceptable at high frequencies [8].

These problems can often be circumvented by using a smooth, finite approximation of the Dirac distribution, rather than attempting to solve the problem with the actual boundary condition. Such approximations have an additional advantage. 
There exist very few natural systems for which the Dirac distribution is an exact model. For example, the disk heated by a flame would be more accurately modeled by imposing a smooth yet concentrated boundary condition. Thus approximating the Dirac distribution by a smooth function may actually be a better representation of the system under consideration, especially if the width of the approximating function can be coupled to the physics of the problem.

A suitable approximating function which is convenient for computation should satisfy the following properties everywhere on the domain under consideration:

1. Its limit with some defining parameter is the Dirac distribution;

2. It is positive, decreases monotonically from a finite maximum at the source point, and tends to zero at the domain extremes;

3. Its derivative exists and is continuous;

4. It is symmetric about the source point;

5. It is representable by a reasonably simple Fourier integral (for infinite domains) or Fourier series (for finite domains).

\section{The Gaussian as an Approximation of the Dirac Distribution}

The Gaussian distribution with zero mean and standard deviation $\sigma$ is given by:

$$
\Phi(\theta)=\frac{a}{\sqrt{\pi}} e^{-\left(\theta^{\prime} \sigma\right)^{2}} .
$$

This distribution, when normalized, can be used to approximate the Dirac distribution by requiring that the amplitude becomes large at the same rate at which the standard deviation becomes small:

$$
\phi(\theta)=\frac{\varepsilon}{\sqrt{\pi}} e^{-(\varepsilon t)^{2}}
$$

To see this, first note that for $\theta=0$ the value of the Gaussian is

$$
\phi(0)=\varepsilon / \sqrt{\pi}
$$

which approaches infinity with $\varepsilon$. For $\theta \neq 0$ the limit becomes (using l'Hôpital's rule):

$$
\begin{aligned}
\left.\lim _{\varepsilon \rightarrow \infty} \phi(\theta)\right|_{\theta \neq 0} & =\frac{1}{\sqrt{\pi}} \lim _{\varepsilon \rightarrow \infty} \frac{\varepsilon}{e^{\varepsilon^{2} \theta^{2}}} \\
& =\frac{1}{\sqrt{\pi}} \lim _{\varepsilon \rightarrow \infty} \frac{1}{2 \theta^{2} \varepsilon e^{\varepsilon^{2} \theta^{2}}} \\
& =0 .
\end{aligned}
$$


It can be shown that, since the distribution has been normalized, Eq. (5) is satisfied, and thus the Gaussian is equivalent to the Dirac distribution in the limit $\vdots \rightarrow \infty$.

\section{The Fourier Transform of the Gaussian on an Infintte Domain}

With the Fourier transform pair defined as

$$
\begin{aligned}
& f(\omega) \triangleq \int_{t=-\infty}^{\infty} f(t) e^{-i m t} d t \\
& f(t) \triangleq \frac{1}{2 \pi} \int_{\omega=-\infty}^{x} f(\omega) e^{2 \omega t} d \omega
\end{aligned}
$$

the Fourier transform of the Dirac distribution is [7]

$$
\tilde{\delta}(\omega)=\int_{-\infty}^{\infty} \delta_{0}(t) e^{-i \omega t} d t=1
$$

It can be seen that, for acceptable results at high frequencies, an extensive time-history of the solution must be provided, since the Fourier transform of the Dirac distribution contains all frequencies with equal amplitude [8]. If frequency information is to be recovered from a time simulation, it may be impracticai to compute the solution for a sufficient number of time steps, and the resulting spectrum will be meaningless at high frequencies.

Since the Gaussian, $\phi(t)$, is smooth, it is not as subject to the high-frequency degradation. Its transform is [8]

$$
\bar{\phi}(\omega)=\int_{-\infty}^{\infty} \frac{c e^{-\left(\varepsilon^{2} t^{2}+i \omega t\right)}}{\sqrt{\pi}} d t=e^{-i \omega \cdot(2 \varepsilon))^{2}} .
$$

Note that, as required, the integral of this quantity over the $\omega$-domain is unity.

\section{The Fourifr Transform of the $\beta_{\pi}$-Distribution on a Finite Domain}

The Gaussian has an important shortcoming for the current application as an approximation of the Dirac distribution on a finite domain, namely that the first derivative of the resulting approximation is discontinuous at $\theta= \pm \pi$. Another approximation is desired.

An approximating function satisfying all of the properties 1 through 5 in Section 1 above is provided by the $\beta$-distribution of probability theory [12]. This distribution is normally defined on the interval $x \in[0,1]$. To accommodate the 
domain under consideration, $x \in[-\pi, \pi]$, shift the coordinate axis and renormalize by dividing by $2 \pi$, so that the area under the distribution is unity. This new distribution, denoted as $\beta_{\pi}(\theta)$, is given by

$$
\beta_{\pi}(\theta) \triangleq \begin{cases}\frac{(\pi+\theta)^{a-1}(\pi-\theta)^{b-1}}{(2 \pi)^{2 a-1} B(a, b)} & \forall \theta \in[-\pi, \pi] \\ 0 & \text { otherwise. }\end{cases}
$$

$B(a, b)$ is the well-known $B$-function [1, formula $(6.2 .2)]$,

$$
\begin{aligned}
B(a, b) & \triangleq \int_{\theta=-\pi}^{\pi}(\pi+\theta)^{a-1}(\pi-\theta)^{b-1} d \theta \\
& =\frac{\Gamma(a) \Gamma(b)}{\Gamma(a+b)}
\end{aligned}
$$

where $\Gamma(x)$ is the familiar Gamma function, discussed in [1].

For the current application, the distribution should be symmetrical, so that $b=a$, or

$$
\beta_{\pi}(\theta)=\frac{(2 \pi)^{1-2 a}\left(\pi^{2}-\theta^{2}\right)^{a-1}}{B(a, a)}
$$

Additionally, to avoid integration problems in the following development, it is required that $a>2$, which is not a serious restriction, since $a$ should be large if an approximation of the Dirac distribution is required. Because of the normalization, the integral of the distribution over the domain is unity. The following limiting values are easily verified:

$$
\begin{aligned}
\lim _{\theta \rightarrow 0} \beta_{\pi} & =\left[2^{1-2 a} /(\pi B(a, a))\right]^{-} \\
d \beta_{\pi} / d \theta & =0^{\mp} \\
\lim _{\theta \rightarrow+\pi^{\mp}} \beta_{\pi} & =0^{+} \\
d \beta_{\pi} / d \theta & =0^{\mp} .
\end{aligned}
$$

Since the derivatives approach zero as $\theta \rightarrow \pm \pi$ from the domain interior, they are continuous there. The $\beta_{\pi}$-distribution approaches the Dirac distribution when $a \rightarrow \infty$ in Eq. (16), as will be shown.

The Fourier coefficients can now be determined. Again, the odd coefficients, $b_{n}$, are all zero while the even coefficients are evaluated as

$$
a_{n}=\frac{1}{\pi} \int_{\theta=-\pi}^{\pi} \beta_{\pi} \cos (n \theta) d \theta \text {. }
$$




\subsection{Fourier-Bessel Series}

While it is mathematically possible to determine the Fourier coefficients for the $\beta_{\pi}$-distribution using a Taylor series, such a series is not computationally useful for large $n$, since the number of required Fourier terms increases with $a$ for resolution of the peak. It is apparent that the Taylor series cannot be truncated until the Taylor index, $m$, is much greater than the Fourier index, $n$, requiring computation: of large exponentials. It is thus fortuitous that the integral appearing in Eq. (17) can be evaluated directly using $[6$, integral $3.771(8)]$ :

$$
\int_{\theta=0}^{n}\left(\pi^{2}-\theta^{2}\right)^{a-1} \cos (n \theta) d \theta=\frac{\sqrt{\pi}}{2}\left(\frac{2 \pi}{n}\right)^{a-1} \Gamma(a) J_{a-12}(\pi n)
$$

where $J_{v}(x)$ is the Bessel function of the first kind of order $\forall$. Thus the $n$th Fourie: coefficient of the symmetric $\beta_{\pi}$-distribution is given by

$$
a_{n}=\frac{1}{2 \pi^{3.2}} e^{E_{n}}
$$

where

$$
\begin{aligned}
E_{n} \triangleq & \left(\frac{1}{2}-a\right) \ln n+\left(\frac{3}{2}-a\right) \ln 2 \pi \\
& +\ln \Gamma(2 a)-\ln \Gamma(a) \\
& -\ln J_{a-1: 2}(\pi n) .
\end{aligned}
$$

Computing the $\beta_{\pi}$-distribution or its Fourier coefincients for large order $a$ necessitates computing $\Gamma(a)$ and $J_{v}(x)$ over large ranges of both $v=a-\frac{1}{2}$ and $x=\pi n$. An excellent technical discussion and FORTRAN code for computing $y(x)$ and $\Gamma(a)$ for all ranges of integer $n$ and $x$ are provided by [11]. However, for even moderate values of $a$, these programs result in underflow or overflow errors. slight modifications they were used to directly compute $\ln J_{i l}(x)$ and $\ln \Gamma(a)$ ingread. For successful computation, the form of Eq. (19) is crucial. In particular, $E_{n}$ mlst be computed in its entirety before substitution into (19) to avoid underflow or overflow errors on even the largest computers. Similarly, Eg, (16) is evaluated using

$$
\beta_{\pi}(\theta)=(2 \pi)^{1-2 a} e^{t_{0}}
$$

where

$$
E_{\theta} \triangleq(a-1) \ln \left(\pi^{2}-\theta^{2}\right)+\ln \Gamma(2 a)-2 \ln \Gamma(a)
$$

Tables I and II in the appendix present the first several Fourier coefficients of the $\beta_{\pi}$-distribution for various values of $a$. 


\subsection{Asymptotic Limit}

$J_{v}(x)$ is given asymptotically with large $v$ as [1, Eq. (9.3.1)]

$$
J_{v}(x) \sim \frac{1}{\sqrt{2 \pi v}}\left(\frac{e x}{2 v}\right)^{v}
$$

or

$$
J_{a-1,2}(\pi n) \sim \frac{1}{\sqrt{\pi(2 a-1)}}\left(\frac{e \pi n}{2 a-1}\right)^{a-1 / 2} .
$$

Substituting (24) in (19) via (20) and cancelling terms yields

$$
a_{n}=\frac{e^{m} \Gamma(2 m+1)}{\pi 2^{2 m+1 / 2} m^{m+1 / 2} \Gamma(m+1 / 2)}
$$

where

$$
m \triangleq a-\frac{1}{2}, \quad \text { for large values of } a
$$

which is independent of $n$. This shows that the $\beta_{\pi}$-distribution approaches the Dirac distribution as the order $a$ approaches $\infty$. Indeed, substituting the duplication formula [1, Eq. (6.1.18)],

$$
\Gamma(2 m)=(2 \pi)^{-1 / 2} 2^{2 m-1 / 2} \Gamma(m) \Gamma\left(m+\frac{1}{2}\right),
$$

the recurrence relation $[10]$,

$$
\Gamma(m+1)=m \Gamma(m)
$$

and Stirling's formula [10],

$$
\Gamma(x)=\sqrt{\frac{2 \pi}{x}} x^{x} e^{-x}[1+r(x)],
$$

gives

$$
\lim _{m \rightarrow \infty} a_{n}=\lim _{m \rightarrow \infty} \frac{2 m+1}{2 \pi m}=\frac{1}{\pi}
$$

or

$$
\lim _{a \rightarrow \infty} \beta_{\pi}(\theta)=\delta_{0}(\theta)
$$

Of course, this asymptotic form is not helpful for the computation, since it returns the Fourier coefficients of the Dirac distribution. 


\section{SOME COMPARISONS}

Figure 1 presents a comparison of the truncated Fourier representations of the Dirac and $\beta_{\pi}$-distributions on the interval $\theta \in[-\pi, \pi]$. The order of the $\beta_{\pi}$-distribution, $a$, has been taken as 100.5 and the Fourier series has beer truncated at five terms. The Fourier representation of the $\beta_{\pi}$-distribution for this figure was generated using Eq. (19). It can be seen from this figure that, while the truncated Fourier representation of the Dirac distribution is associated with an increasing amplitude of high-frequency oscillations (a Gibbs phenomenon) neat the source point, the $\beta_{\pi}$-distribution is very smooth. Since the $\beta_{n}$-distribution is continuous on the closed domain, its Fourier series is uniformiy convergent there.

Figure 2 shows the values of some of the Fourier coefficients, $a_{n}$, of the $\beta_{\pi}$-distribution (as computed using Eq. (19)) for various values of the order $a$. (Those of the Dirac distribution, which are all $a_{n}=1 / \pi$, are not shown.) It can be seen that, as $a$ is increased, the coefficients lie on curves which are increasingly flat near the origin and tail off more and more gently. The value of $a_{0}$ is $1 / \pi$ for all values of the order, $a$.

The coefficients used to produce Fig. 2 are listed in the tables included in the Appendix. For large values of $a$ these are slowly decreasing with Fourier index, $n$. This does not pose any computational difficulties since the truncation error in summing the series is less than the magnitude of the last coefficient included. Thus, for a taking values of $100.5,1000.5$, and $10,000.5$, the truncation error will be less than $2 \times 10^{-5}$, provided the respective series include at least 20.63 , and $199 \mathrm{com}$ ponents. Summing such a large series is not difficult with even a desktop computer. In any case, the slow convergence of the proposed $\beta_{\pi}$-distribution is to be expectec. since it is approximating a series with non-uniform convergence which does not

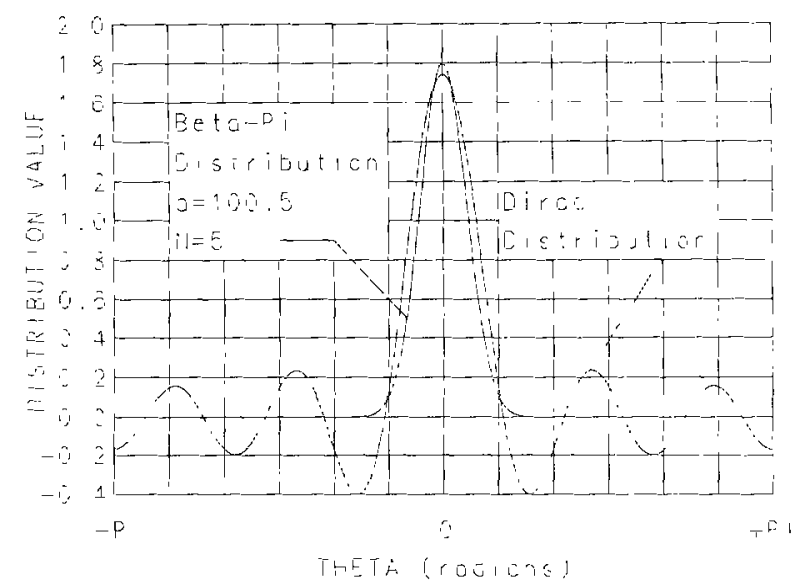

Fig. 1. Truncated Fourier representations of the Dirac and $\beta_{\pi}$-distributions. 


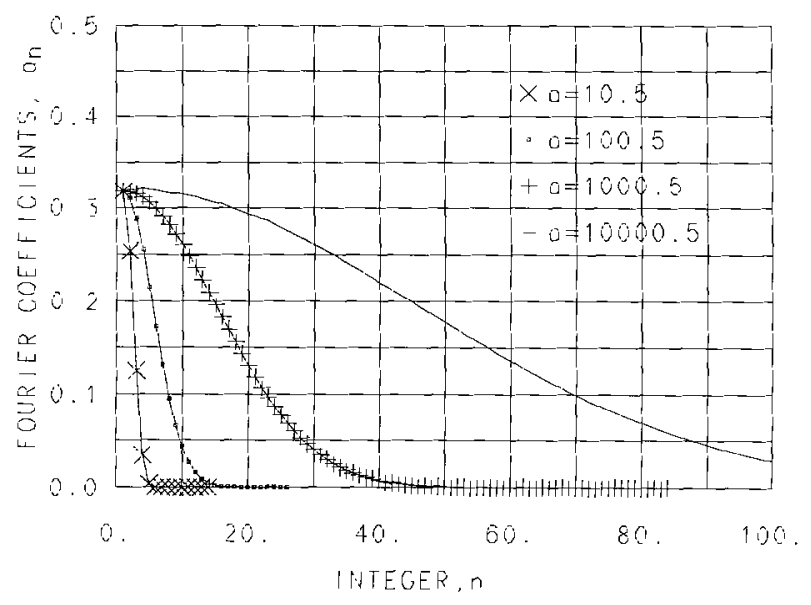

FIG. 2. Some Fourier coefficients of the $\beta_{\pi}$-distribution. All Fourier coefficients of the Dirac distribution are $1 / \pi$.

converge at all at the singular point. Figures 3 and 5 illustrate that useful results reflecting negligible truncation error can be easily generated using the $\beta_{\pi}$-distribution.

Figure 3 shows Fourier representations of the $\beta_{\pi}$-distribution with the order $a$ chosen as $10.5,100.5,1000.5$, and $10,000.5$. Notice that each curve is smooth. The truncation indices of the Fourier coefficients retained in generating each of these curves were, respectively, $4,12,42$, and 130.

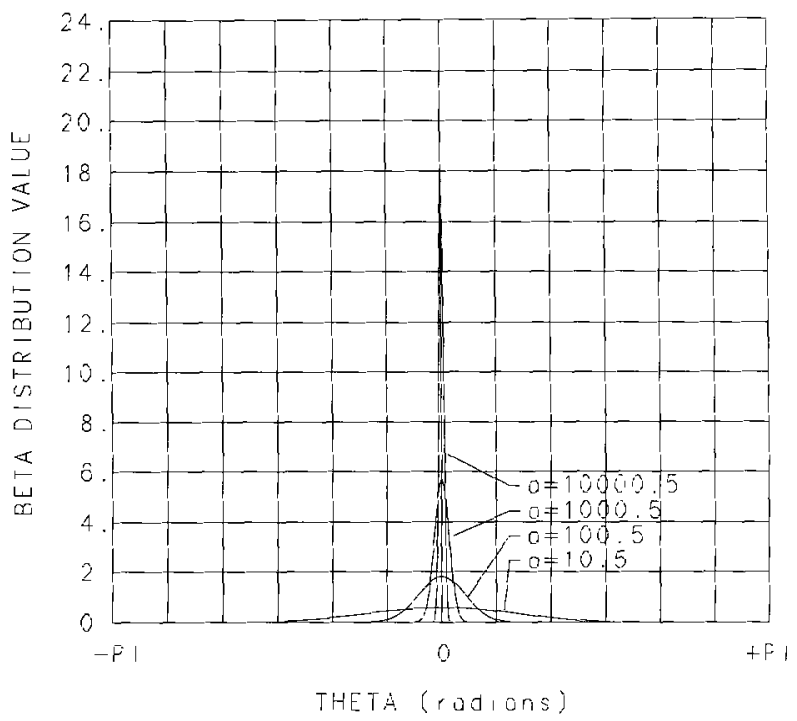

Fig. 3. Fourier representations of the $\beta_{\pi}$-distribution with four values of the order $a$. 


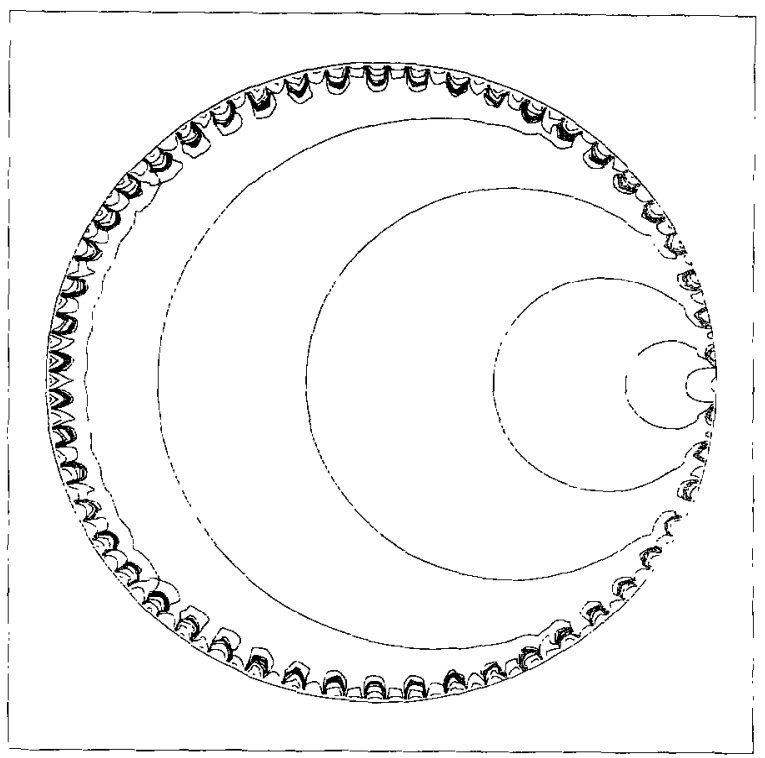

Fig. 4. Soiution of the steady heat equation in a disk with a singularity on the boundary, represented by the Fourier series of the Dirac-distribution truncated at index $N=50$.

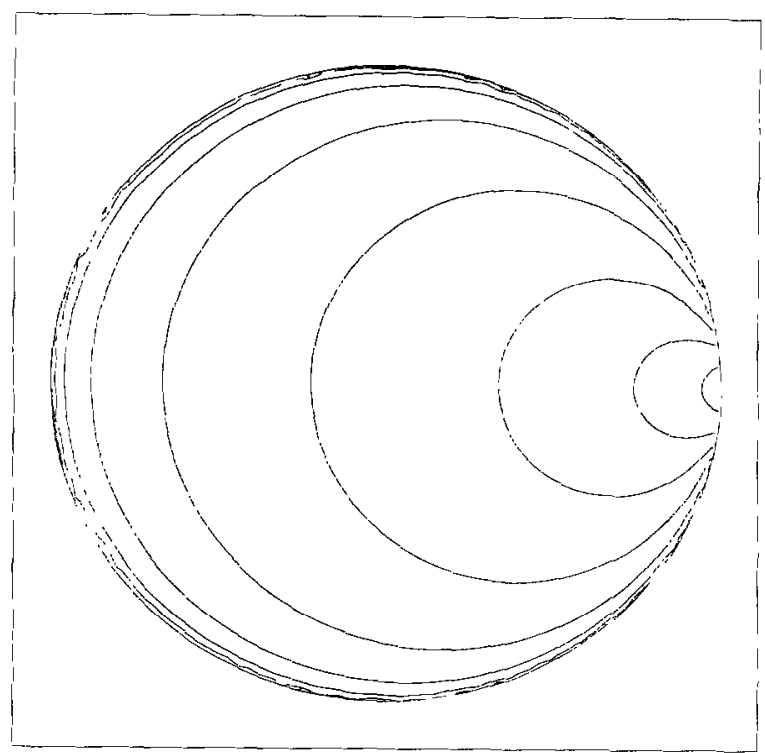

FiG. 5. Solution of the steady heat equation in a disk with a singuiarity on the boundary, represented by the Fourier series of the $\beta_{\pi}$-distribution of order $a=1000.5$ truncated at index $N=50$ ). 
Figures 4 and 5 show the results of the problem outlined in the Introduction: the solution of the steady heat equation in a disk with a singularity in the boundary condition. Figure 4 represents the solution computed using the truncated Fourier representation of the Dirac distribution. Notice that the Gibbs phenomenon in the boundary condition contaminates the solution, causing unrealistic jaggedness near the boundary, especially in the neighborhood of the source point. This is an artifact of the non-uniform convergence of the series representing the boundary condition. The equivalent results computed using the $\beta_{\pi}$-distribution are well behaved, as is evident from Fig. 5, which was computed using $a=1000.5$. While the error is (of course) still infinite at the source point, the solutions are uniformly convergent everywhere else in the domain and on the boundary. The index of the highest Fourier coefficient retained in generating each of these figures was 50.

\section{CONCLUSION}

It has been suggested that the troublesome convergence associated with the Fourier representation of the Dirac distribution can be avoided by replacing the singular distribution with a smooth approximation. A previously known approximation for the infinite domain, the Gaussian, has been presented. A new approximation, the $\beta_{\pi}$-distribution, has herein been developed for the finite domain, and procedures have been given for computing its Fourier coefficients. The great advantage of the $\beta_{\pi}$-distribution is that it provides continuity of all derivatives everywhere in the finite domain, including the endpoints.

The procedure for computing the Fouricr cocfficients of the $\beta_{\pi}$-distribution involves the Fourier-Bessel decomposition of a known integral. It has been demonstrated that existing numerical techniques are capable of evaluating the truncated Fourier representation of the $\beta_{\pi}$-distribution for very large values of both the order, $a$, and the truncation index, $N$. Tables of coefficients of the distribution for various values of the order are presented in the Appendix.

The truncated Fourier representations of the $\beta_{\pi}$-and the Dirac distributions have been compared for various values of the order and truncation index. Finally, the results have been employed in comparing the solution of the stcady heat cquation in a disk as computed using each of the representations. It is shown that the Gibbs phenomenon associated with the Dirac representation is avoided by using the proposed approximation.

It has been pointed out that singular distributions are often idealizations of smooth (albeit very peaked) functions and that using a smooth approximation may be more representative of the physics under analysis. Guidelines for choosing the peakedness of the approximating distribution could be derived from analysis of the physics underlying the problem being studied. If such an analysis indicated that the physical distribution was non-symmetric, techniques similar to those proposed herein could be applied, since the $\beta_{\pi}$-distribution need not be symmetric. 


\section{APpendiX: Tabulated Fourier Coefficients of the $\beta_{\pi}$-Distribution FOR VARIOUS VALUES OF THE ORDER $a$}

The following tables were generated using Eq. (18) through (20). With these coefficients, the $\beta_{\pi}$-distribution is represented by its truncated Fouricr scrics using the formula:

$$
\beta_{\pi}(\theta) \sim \frac{a_{0}}{2}+\sum_{n=0}^{N}\left[a_{n} \cos (n \theta)+b_{n} \sin (n \theta)\right]
$$

TABLE I

Fourier Coefficients for the $\beta_{\pi}$-Distribution for Three Values of the Order $a$

\begin{tabular}{|c|c|c|c|c|c|c|}
\hline \multirow[t]{2}{*}{7} & \multicolumn{3}{|c|}{$a_{n}$} & \multirow[t]{2}{*}{$n$} & \multicolumn{2}{|c|}{$x_{n}$} \\
\hline & $a=100.5$ & $a=1000.5$ & $a=10000.5$ & & $a=1000.5$ & $a=10000.5$ \\
\hline 0 & $3.18310 \mathrm{E}-01$ & $3.18310 \mathrm{E}-01$ & $3.18310 \mathrm{E}-01$ & & & \\
\hline 1 & $3.10629 \mathrm{E}-01$ & $3.17530 \mathrm{E}-1$ & $3.20216 \mathrm{E}-1$ & 51 & $5.12350 \mathrm{E}-04$ & $1.69402 \mathrm{E}-01$ \\
\hline 2 & $2.8 B 6 \epsilon 5 \mathrm{E}-01$ & $3.15175 \mathrm{E}-01$ & $3.19435 \mathrm{E}-01$ & 52 & $3.96736 \mathrm{E}-04$ & $164130 \mathrm{E}-01$ \\
\hline 3 & $2.55423 \mathrm{E}-01$ & $3.11275 \mathrm{E}-01$ & $3.21156 \mathrm{E}-01$ & 53 & $3.05751 \mathrm{E}-04$ & $1.60387 \mathrm{E}-01$ \\
\hline 4 & $2.15167 \mathrm{E}-01$ & 3.06038E-01 & 3.19904E-01 & 54 & $2.34428 \mathrm{E}-04$ & $1.56061 \mathrm{E}-01$ \\
\hline 5 & $1.72509 \mathbf{E}-01$ & $2.99277 \mathrm{E}-01$ & $3.19279 \mathrm{E}-01$ & 55 & $1.78866 \mathrm{E}-04$ & $1.52445 \mathrm{E}-01$ \\
\hline 6 & $1.31595 \mathrm{E}-01$ & $2.91275 \mathrm{E}-01$ & 3.17724E-01 & 56 & $135742 \mathrm{E}-04$ & $1.48334 \mathrm{E}-01$ \\
\hline 7 & $9.54739 \mathrm{E}-02$ & $2.82073 \mathrm{E}-01$ & $3.16485 \mathrm{E}-01$ & 57 & $1.02489 \mathrm{E}-04$ & $143770 \mathrm{E}-01$ \\
\hline 8 & $6 \mathrm{58454E-02}$ & 2.71863E-01 & $3.16485 \mathrm{E}-01$ & 58 & $7.70235 \mathrm{E}-05$ & $1.39892 \mathrm{E} \cdot 01$ \\
\hline 9 & $4.31436 \mathrm{~F}-02$ & $2.60747 \mathrm{E}-01$ & $3.14637 \mathrm{E}-01$ & 59 & 3.75965E-05 & $1.36118 \mathrm{E}-01$ \\
\hline 10 & $2.68392 \mathrm{E}-02$ & $2.48807 \mathrm{E}-01$ & $3.13410 \mathrm{E}-01$ & 60 & $4.28386 \mathrm{E}-05$ & $1.32447 \mathrm{E}-01$ \\
\hline 21 & $1.58393 \mathrm{E} 02$ & $2.36257 \mathrm{E}-01$ & $3.12188 \mathrm{E}-01$ & 61 & $3.17070 \mathrm{E}-05$ & $1.27871 \mathrm{E}-01$ \\
\hline 12 & $8.86002 \mathrm{E}-03$ & $2.23192 \mathrm{E}-01$ & $3.09759 \mathrm{E}-01$ & 62 & $233422 \mathrm{E}-05$ & 1.23937E-01 \\
\hline 13 & $4.69276 \mathrm{E}-03$ & $2.09875 \mathrm{E}-01$ & 3.07949E-D1 & 63 & $1.70933 \mathrm{E}-05$ & $120594 \mathrm{E}-01$ \\
\hline 14 & 2.35093E-03 & $1.96342 \mathrm{E}-01$ & 3.06150E-01 & 64 & $1.24667 \mathrm{E}-05$ & $116884 \mathrm{E}-01$ \\
\hline 15 & $1.11241 \mathrm{E}_{-03}$ & $1.82788 \mathrm{E}-01$ & $304361 \mathrm{E}-01$ & 65 & $903886 \mathrm{E}-06$ & 1. $13288 \mathrm{E}-01$ \\
\hline 16 & $4.96467 \mathrm{E}-04$ & $1.69382 \mathrm{E}-01$ & $3.01403 \mathrm{E}-01$ & 66 & $6.52476 \mathrm{E}-06$ & $1.09802 \mathrm{E}-0:$ \\
\hline 17 & $208631 \mathrm{E}-04$ & $1.56080 \mathrm{E}-01$ & 2.98474E-01 & 67 & $4.58243 \mathrm{E}-06$ & $106424 \mathrm{E}-01$ \\
\hline 18 & $823959 \mathrm{E}-05$ & $1.43192 \mathrm{E}-01$ & $2.96151 \mathrm{E}-01$ & 68 & $3.34311 \mathrm{E}-06$ & $1.02347 \mathrm{E}-01$ \\
\hline 19 & $3.05162 \mathrm{E}-05$ & 1.30664E-01 & $2.93273 \mathrm{E}-01$ & 69 & $2.37700 \mathrm{E}-06$ & $9.91984 E-02$ \\
\hline 20 & $1.05728 \mathrm{E}-05$ & $1.18710 \mathrm{E}-01$ & $2.91560 \mathrm{E}-01$ & 70 & 1.67979E-06 & $561464 \mathrm{E}-02$ \\
\hline 21 & $3.41677 \mathrm{E}-06$ & $1.07298 \mathrm{E}-01$ & $2.88163 \mathrm{E}-01$ & 71 & i. $18159 \mathrm{E}-06$ & $9.24631 \mathrm{E}-02$ \\
\hline 22 & $1.02656 \mathrm{E}-06$ & $9.64874 \mathrm{E}-02$ & 2.85921E-01 & 72 & $8.27104 \mathrm{E}-07$ & $8.96183 \mathrm{E}-02$ \\
\hline 23 & 2.85613E-07 & 8.63641E-02 & $2.82038 \mathrm{E}-01$ & 73 & $5.75722 \mathrm{E}-07$ & 8.65224E-02 \\
\hline 24 & & $768389 \mathrm{E}-02$ & 2.78752E-01 & 74 & $3.98645 \mathrm{E}-0 ?$ & $832073 \mathrm{E}-02$ \\
\hline 25 & & $6.81198 \mathrm{E}-02$ & $2.75505 \mathrm{E}-\mathrm{Gl}$ & $7 \vec{s}$ & $2.74755 \mathrm{E}-07$ & 8.03333E-02 \\
\hline 26 & & $6.00568 \mathrm{E}-02$ & $2.71764 \mathrm{E}-01$ & 76 & $1.88260 \mathrm{E}-07$ & 7.72558E-02 \\
\hline 27 & & $5.27032 \mathrm{E}-02$ & $2.68598 \mathrm{E}-01$ & 77 & 1.23429E-07 & 7. $42962 \mathrm{E}-02$ \\
\hline 28 & & $4.60022 \mathrm{E}-02$ & $2.64433 \mathrm{E}-01$ & 78 & & $717296 \mathrm{E}-02$ \\
\hline 29 & & $3.99674 \mathrm{E}-02$ & $2.60334 \mathrm{E}-01$ & 79 & & $5.8981 \mathrm{TE}-02$ \\
\hline 30 & & $3.45383 \mathrm{E}-02$ & $2.57301 \mathrm{E}-01$ & 80 & & $6.53390 \mathrm{E}-02$ \\
\hline 31 & & $\overline{2} .97121 \mathrm{E}-02$ & $\overline{2} 5 \overline{3} 312 \mathrm{E} 0 \overline{1}$ & 81 & & $6 . \overline{37976 \mathrm{E}-12}$ \\
\hline 32 & & $2.54327 \mathrm{E}-02$ & $2.50361 \mathrm{E}-01$ & 82 & & $6.11144 \mathrm{E}-02$ \\
\hline 33 & & $2.16557 \mathrm{E}-02$ & $2.45518 \mathrm{E}-01$ & 83 & & 5.87731 E-C2 \\
\hline 34 & & $1.83453 \mathrm{E}_{-02}$ & $2.40769 \mathrm{E}-01$ & 84 & & $5.63012 \mathrm{E}-02$ \\
\hline 35 & & $1.54729 \mathrm{E}-02$ & $2.37037 \mathrm{E}-01$ & 85 & & $5.41443 \mathrm{E}-02$ \\
\hline 36 & & $1.29802 \mathrm{E}-02$ & $2.33362 \mathrm{E}-01$ & 86 & & $5.16649 \mathrm{E}-02$ \\
\hline 37 & & $108361 \mathrm{E}-02$ & $2.28848 \mathrm{E}-01$ & 87 & & $4.94919 \mathrm{E}-02$ \\
\hline 38 & & $8.99996 \mathrm{E}-03$ & $2.25300 \mathrm{E}_{-01}$ & 88 & & $4.75959 \mathrm{E} 02$ \\
\hline 39 & & $7.44031 \mathrm{E}-03$ & $2.20081 \mathrm{E}-01$ & 89 & & $455941 \mathrm{E}-02$ \\
\hline 40 & & $6.12023 \mathrm{E}-03$ & $2.16669 \mathrm{E}-01$ & 90 & & $4.35912 \mathrm{E}-02$ \\
\hline 41 & & $5.00678 \mathrm{E}-03$ & $2.11650 \mathrm{E}-01$ & 91 & & $4.15950 \mathrm{E}-02$ \\
\hline 42 & & $4.07795 \mathrm{E}-03$ & $2.07556 \mathrm{~F}-01$ & 02 & & $398456 F_{-02}$ \\
\hline 43 & & 3.30363E-03 & $2.03542 \mathrm{E}-01$ & 93 & & $378727 \mathrm{E}-02$ \\
\hline 44 & & $2.66363 \mathrm{E}-03$ & $1.99605 \mathrm{E}-01$ & 94 & & $363508 \mathrm{E}-02$ \\
\hline 45 & & $2.13637 \mathrm{E}-03$ & $1.94221 \mathrm{E}-01$ & 95 & & $3.45509 \mathrm{E}-02$ \\
\hline 46 & & $1.70534 \mathrm{E}-03$ & $1.90464 \mathrm{E}-01$ & 96 & & $3.31524 \mathrm{E}-02$ \\
\hline 47 & & $1.35415 \mathrm{E}-03$ & $1.86052 \mathrm{E}-01$ & 97 & & 3 $14390 \mathrm{E}-02$ \\
\hline 48 & & $1.07017 \mathrm{E}-03$ & $1.81742 \mathrm{E}-01$ & 98 & & $3.00770 E-02$ \\
\hline 49 & & $841224 \mathrm{E}-04$ & 1.77532E-01 & 99 & & $2.86437 \mathrm{E}-02$ \\
\hline 30 & & $6.58111 \mathrm{E}-04$ & $1.73420 \mathrm{E}-01$ & 100 & & $272787 \mathrm{E}-02$ \\
\hline
\end{tabular}


TABLE II

Fourier Coefficients for the $\beta_{\pi}$-Distribution for Order, $a=10,000.5$ starting with Index $n=101$

\begin{tabular}{|c|c|c|c|c|c|c|c|c|c|}
\hline $\bar{n}$ & $a_{n}$ & $n$ & $\overline{a_{n}}$ & $n$ & $a_{n}$ & $\bar{n}$ & $a_{n}$ & $n$ & $\overline{a_{n}}$ \\
\hline 101 & $2.59280 \mathrm{E}-02$ & 121 & $8.66786 \mathrm{E}^{-03}$ & 141 & $2.38358 \mathrm{E}-03$ & 161 & $5.33932 \mathrm{E}-04$ & 181 & $9.87674 \mathrm{E}-05$ \\
\hline 102 & 2.47407E-02 & 122 & $8.17457 \mathrm{E}-03$ & 142 & $2.21308 \mathrm{E}-03$ & 162 & $4.94771 \mathrm{E}-04$ & 182 & $9.02807 \mathrm{E}-05$ \\
\hline 103 & $2.34240 \mathrm{E}-02$ & 123 & $7.66431 \mathrm{E}-03$ & 143 & $2.06282 \mathrm{E}-03$ & 163 & $4.55805 E-04$ & 183 & $8.26846 \mathrm{E}-05$ \\
\hline 104 & $2.23077 \mathrm{E}-02$ & 124 & $7.19996 \mathrm{E}-03$ & 144 & $1.92652 \mathrm{E}-03$ & 164 & $4.19907 \mathrm{E}-04$ & 184 & $7.57276 \mathrm{E}-05$ \\
\hline 105 & $2.11618 \mathrm{E}-02$ & 125 & $6.81678 \mathrm{E}-03$ & 145 & $1.79221 \mathrm{E}-03$ & 165 & $3.89110 \mathrm{E}-04$ & 185 & $6.89508 \mathrm{E}-05$ \\
\hline 106 & $2.01140 \mathrm{E}-02$ & 126 & $6.39128 \mathrm{E}-03$ & 146 & $1.67052 \mathrm{E}-03$ & 166 & 3.57765E-04 & 186 & $6.27804 \mathrm{E}-05$ \\
\hline 107 & 1.90808E-02 & 127 & $6.01579 E-03$ & 147 & $1.55103 \mathrm{E}-03$ & 167 & 3.30233E-04 & 187 & $5.74981 \mathrm{E}-05$ \\
\hline 108 & $1.81007 \mathrm{E}-02$ & 128 & $5.65131 \mathrm{E}-03$ & 148 & $1.44289 \mathrm{E}-03$ & 168 & 3.03631E-04 & 188 & $5.22505 \mathrm{E}-05$ \\
\hline 109 & $1.71039 \mathrm{E}-02$ & 129 & $5.29856 \mathrm{E}-03$ & 149 & $1.33446 \mathrm{E}-03$ & 169 & $2.79172 \mathrm{E}-04$ & 189 & $4.75746 \mathrm{E}-05$ \\
\hline 110 & $1.62253 \mathrm{E}-02$ & 130 & $4.97753 \mathrm{E}-03$ & 150 & $1.24872 \mathrm{E}-03$ & 170 & $2.56684 \mathrm{E}-04$ & 190 & $4.32326 \mathrm{E}-05$ \\
\hline 111 & $1.54219 \mathrm{E}-02$ & 131 & $4.66683 \overline{\mathrm{E}}-03$ & 151 & $1.15488 \mathrm{E}-03$ & 171 & $2.35087 \mathrm{E}-04$ & 191 & $3.95178 \mathrm{E}-05$ \\
\hline 112 & $1.45159 \mathrm{E}-02$ & 132 & $4.36699 \mathrm{E}-03$ & 152 & $1.07017 \mathrm{E}-03$ & 172 & $2.16995 \mathrm{E}-04$ & 192 & $3.59814 \mathrm{E}-05$ \\
\hline 113 & 1.37702E-02 & 133 & $4.08642 \mathrm{E}-03$ & 153 & $9.97516 \mathrm{E}-04$ & 173 & $1.99126 \mathrm{E}-04$ & 193 & $3.25700 \mathrm{E}-05$ \\
\hline 114 & 1.29866E-02 & 134 & $3.83134 \mathrm{E}-03$ & 154 & $9.22551 \mathrm{E}-04$ & 174 & $1.82728 E-04$ & 194 & $2.95975 \mathrm{E}-05$ \\
\hline 115 & $1.23195 \mathrm{E}-02$ & 135 & 3.59219E-03 & 155 & $8.53220 \mathrm{E}-04$ & 175 & $1.67354 \mathrm{E}-04$ & 195 & $2.70015 \mathrm{E}-05$ \\
\hline 116 & $1.15731 \mathrm{E}-02$ & 136 & 3.34829E-03 & 156 & $7.90643 \mathrm{E}-04$ & 176 & $1.54173 \mathrm{E}-04$ & 196 & $2.44893 \mathrm{E}-05$ \\
\hline 117 & $1.09572 \mathrm{E}-02$ & 137 & $3.13316 \mathrm{E}-03$ & 157 & $7.32654 \mathrm{E}-04$ & 177 & $1.41201 \mathrm{E}-04$ & 197 & $2.22108 \mathrm{E}-05$ \\
\hline 118 & $1.03538 \mathrm{E}-02$ & 138 & $2.93186 \mathrm{E}-03$ & 158 & $6.77595 \mathrm{E}-04$ & 178 & $1.29068 \mathrm{E}-04$ & 198 & $2.01443 \mathrm{E}-05$ \\
\hline 119 & $9.74554 \mathrm{E}-03$ & 139 & $2.73814 \mathrm{E}-03$ & 159 & $6.27898 \mathrm{E}-04$ & 179 & $1.18209 \mathrm{E}-04$ & 199 & $1.83058 \mathrm{E}-05$ \\
\hline 120 & $9.20889 \mathrm{E}-03$ & 140 & $2.54724 \mathrm{E}-03$ & 160 & $5.80710 \mathrm{E}-04$ & 180 & $1.08052 \mathrm{E}-04$ & 200 & 1.66027E-05 \\
\hline
\end{tabular}

The tables list the coefficients $a_{n}, n>0$ used to generate Fig. 3. Tables I and II list coefficients for $a-100.5,1000.5$, and $10,000.5$. Table I provides coefficients to precision of the order $10^{-7}$ for $a=100.5$ and 1000.5 , and the first 100 coefficients for $a=10,000.5$. Table II continues the coefficients for $a=10,000.5$ through index $n=200$, for which the precision is of the order $10^{5}$. The zeroeth coefficients all take the value $1 / \pi$, which is the value of all of the Fourier coefficients of the Dirac distribution.

\section{REFERENCES}

1. M. Abramowitz and I. A. Stegun, Handbook of Mathematical Functions, 9th ed. (Dover, New York, 1972).

2. W. H. BeYer ANd S. M. Selby, Standard Mathematical Tables, 24th ed. (CRC Press, Cleveland, 1976).

3. N. G. DE BRUijn, Asymptotic Methods in Analysis (Dover, New York, 1981 ).

4. A. ERdÉLYI, Asymptotic Expansions (Dover, New York, 1956).

5. J. E. Freund, Mathematical Statistics, 2nd ed. (Prentice-Hall, Englewood Cliffs, NJ, 1971).

6. I. S. Gradshteyn ANd I. M. RyshiK, Table of Integrals, Series, and Products, corrected and enlarged edition (Academic Press, New York, 1980).

7. W. Karlan, Advanced Calculus, 3rd ed. (Addison-Wesley, Reading, MA, 1984).

8. B. K. King, Time-Domain Analysis of Wave Exciting Forces on Ships and Bodies, Department of Naval Architecture and Marine Engineering Report, No. 306, University of Michigan, Ann Arbor, 1987.

9. I. N. KIRSCHNER, Seminar in Marine Hydrodynamics, Department of Naval Architecture and Marine Engineering, University of Michigan, Ann Arbor, 1988 (unpublished).

10. N. N. Lebedev, Special Functions and Their Applications, translated from the Russian by Richard A. Silverman (Dover, New York, 1972).

11. W. H. Press, B. P. Flannery, S. A. Teukolsky, and W. T. Vetterling, Numerical Recipes (Cambridge Univ. Press, New York, 1987).

12. S. Ross, A First Course in Probability, 2nd ed. (Macmillan Co., New York, 1984).

13. G. P. Tolstov, Fourier Series, translated from the Russian by Richard A. Silverman (Dover, New York, 1962). 